\title{
Adjuvant Chemotherapy in Patients with Node-Negative Non-Small Cell Lung Cancer with Satellite Pulmonary Nodules in the Same Lobe
}

\author{
Jiyoun Park, M.D., Junghee Lee, M.D., Ph.D., Yeong Jeong Jeon, M.D., Ph.D., Sumin Shin, M.D., \\ Jong Ho Cho, M.D., Ph.D., Hong-Kwan Kim, M.D., Ph.D., Yong Soo Choi, M.D., Ph.D., Jhingook Kim, M.D., Ph.D., \\ Jae III Zo, M.D., Ph.D., Young Mog Shim, M.D., Ph.D. \\ Department of Thoracic and Cardiovascular Surgery, Samsung Medical Center, Sungkyunkwan University School of Medicine, Seoul, Korea
}

\section{ARTICLE INFO}

Received September 23, 2021

Revised November 12, 2021

Accepted November 22, 2021

Corresponding author

Junghee Lee

Tel 82-2-02108091

Fax 82-2-3410-106

E-mail jhts.lee@samsung.com

ORCID

https://orcid.org/0000-0003-0466-7630
Background: According to the eighth TNM (tumor-node-metastasis) staging system, the presence of separate tumor nodules in the same lobe is designated as a T3 descriptor. However, it remains unclear whether adjuvant chemotherapy confers survival advantages in this setting.

Methods: We retrospectively identified 142 pathologic T3NOMO patients with additional pulmonary nodules in the same lobe from a single-institutional database from 2004 to 2019. The main outcomes were overall survival and recurrence-free survival. Multivariable Cox regression was used to identify the benefit of adjuvant chemotherapy while adjusting for other variables.

Results: Sixty-one patients received adjuvant chemotherapy (adjuvant group) and 81 patients did not receive adjuvant therapy after surgery (surgery-only group). There were no demonstrable differences between the 2 groups regarding hospital mortality and postoperative complications, indicating that treatment selection had not significantly occurred. However, the use of adjuvant chemotherapy was associated with improved 5-year overall survival (70\% vs. $59 \%, p=0.006)$ and disease-free survival $(60 \%$ vs. $46 \%, p=0.040)$. A multivariable Cox model demonstrated that adjuvant chemotherapy was associated with a survival advantage (adjusted hazard ratio, $0.54 ; \mathrm{p}<0.001$ ). In exploratory analyses of subgroups, the effect of adjuvant chemotherapy seemed to be insufficient in those with small main tumors $(<4 \mathrm{~cm})$.

Conclusion: Adjuvant chemotherapy was associated with better survival in T3 cancers with an additional tumor nodule in the same lobe. However, the role of adjuvant chemotherapy in patient subgroups with small tumors or those without risk factors should be determined via large studies.

Keywords: Adjuvant chemotherapy, Satellite pulmonary nodule, Lung neoplasms, Survival

\section{Introduction}

Lung cancer is the leading cause of cancer mortality worldwide. Despite its poor prognosis, surgery remains the best opportunity to cure localized non-small-cell lung cancer (NSCLC). However, even after cancer has been completely resected, the risk of recurrence remains substantial. In fact, $36 \%$ of patients with stage I-II NSCLC have recur- rence within 5 years of surgery [1]. The Lung Adjuvant Cisplatin Evaluation meta-analysis [2] based on 5 large cisplatin-based adjuvant trials [3-7] suggested that adjuvant cisplatin-based chemotherapy could yield an overall survival advantage of $5.4 \%$ at 5 years. This survival benefit was evident for node-positive NSCLC and for patients with node-negative tumors that were $4 \mathrm{~cm}$ or larger. As a result, international guidelines from Europe and the United States 
recommend adjuvant chemotherapy for resected stage IIIII NSCLC [8-10].

However, these adjuvant studies did not adequately reflect the variety of oncologic scenarios in node-negative cases despite staging beyond T2 due to the locally advanced tumor status. Although only 1 unplanned subgroup analysis of an adjuvant trial demonstrated a survival benefit of adjuvant therapy in node-negative NSCLC sized $4 \mathrm{~cm}$ or greater [11], evidence supporting the use of adjuvant chemotherapy in other settings is still lacking. One example is the presence of the additional nodules in the same lobe of a small node-negative tumor, which makes cancer greater than stage I, but the optimal management of this subset of NSCLC remains unclear.

In 2016, a retrospective study of 1,013 patients in the United States using the National Cancer Database (NCDB) demonstrated the association of favorable survival outcomes with the use of adjuvant therapy in patients with T3 NSCLC who had separate pulmonary nodules in the same lobe [12]. Their results were maintained in a subcohort of 528 patients with node-negative NSCLC and a satellite pulmonary nodule in the same lobe without locally invasive features. However, since the NCDB is hospital-based rather than population-based, and is therefore not designed to be representative of the US population overall, the generalizability of its data is limited [13]. Furthermore, that study was limited in terms of detailed information on tumor characteristics, treatment, and surveillance, such as the diagnosis of satellite pulmonary nodules, the intent of postoperative chemotherapy and agents used therein, and surveillance. These limitations require further studies characterizing the role of adjuvant therapy in this setting with detailed information. Therefore, we aimed to evaluate the effect of adjuvant chemotherapy on survival in patients with nodenegative NSCLC with a satellite pulmonary nodule in the same lobe using a large single-institutional registry with detailed information on the diagnosis and therapeutic variables.

\section{Methods}

\section{Study population}

With the approval of the Institutional Review Board of Samsung Medical Center (no., 2021-08-091), patients with NSCLC with pathologic T3 separate pulmonary nodule in the same lobe who underwent curative-intent upfront surgery between 2004 and $2019(\mathrm{~N}=269)$ were selected from the Registry for Thoracic Cancer Surgery (RTCS) at a sin- gle tertiary-referral academic center. The requirement for informed consent from individual patients was omitted because of the retrospective design of this study. The RTCS includes all patients who have undergone surgery for NSCLC since 1995. The clinical information for these patients is regularly updated by a trained data manager using electronic medical records. Survival information was updated through August 2021 for all patients.

We excluded patients who had direct tumor extension to surrounding structures such as the chest wall, parietal pleura, phrenic nerve, and parietal pericardium $(n=17)$. In addition, we also excluded patients with pathologic nodal disease $(\mathrm{n}=105)$. The final cohort included 142 patients (Fig. 1). We thoroughly reviewed the radiographic appearance along with histological and immunohistochemistry findings within the pathological data to differentiate between double primary lung cancer and a satellite pulmonary nodule. An additional pulmonary nodule is considered to be double primary lung cancer if it differs clearly from the primary tumor in histological type, radiological appearance, or tumor growth rate.

\section{Staging workup and treatment strategy}

The routine preoperative examination included a complete history and physical examination, complete blood counts, chemistry profiles, pulmonary function testing, simple chest radiography, computed tomography (CT) of the chest and upper abdomen, bronchoscopy with washing cytology and/or biopsy, integrated whole-body 18F-fluorodeoxyglucose positron emission tomography and CT (PET/ CT) scans, and brain magnetic resonance imaging. For patients with suspicious nodal involvement, a histopathologic examination through endobronchial ultrasound-guided transbronchial needle aspiration was conducted to rule out nodal metastasis. The surgical procedures included pulmonary resection and systematic lymph node dissection of the ipsilateral hilum and mediastinum.

After surgery, adjuvant chemotherapy was administered at the surgeon's discretion for patients with satellite pulmonary nodules, unless there was an indication for adjuvant therapy, such as pathologic nodal disease or tumor size 4 $\mathrm{cm}$ or greater. Adjuvant chemotherapy was delivered within 2 months of surgical resection, and the chemotherapy regimens comprised 4 cycles of platinum-based doublet agents at 3-week intervals.

Patients were regularly evaluated by CT every 3 to 4 months for the first 2 years following surgery, and then every 6 months thereafter. Patients were evaluated by PET/ 


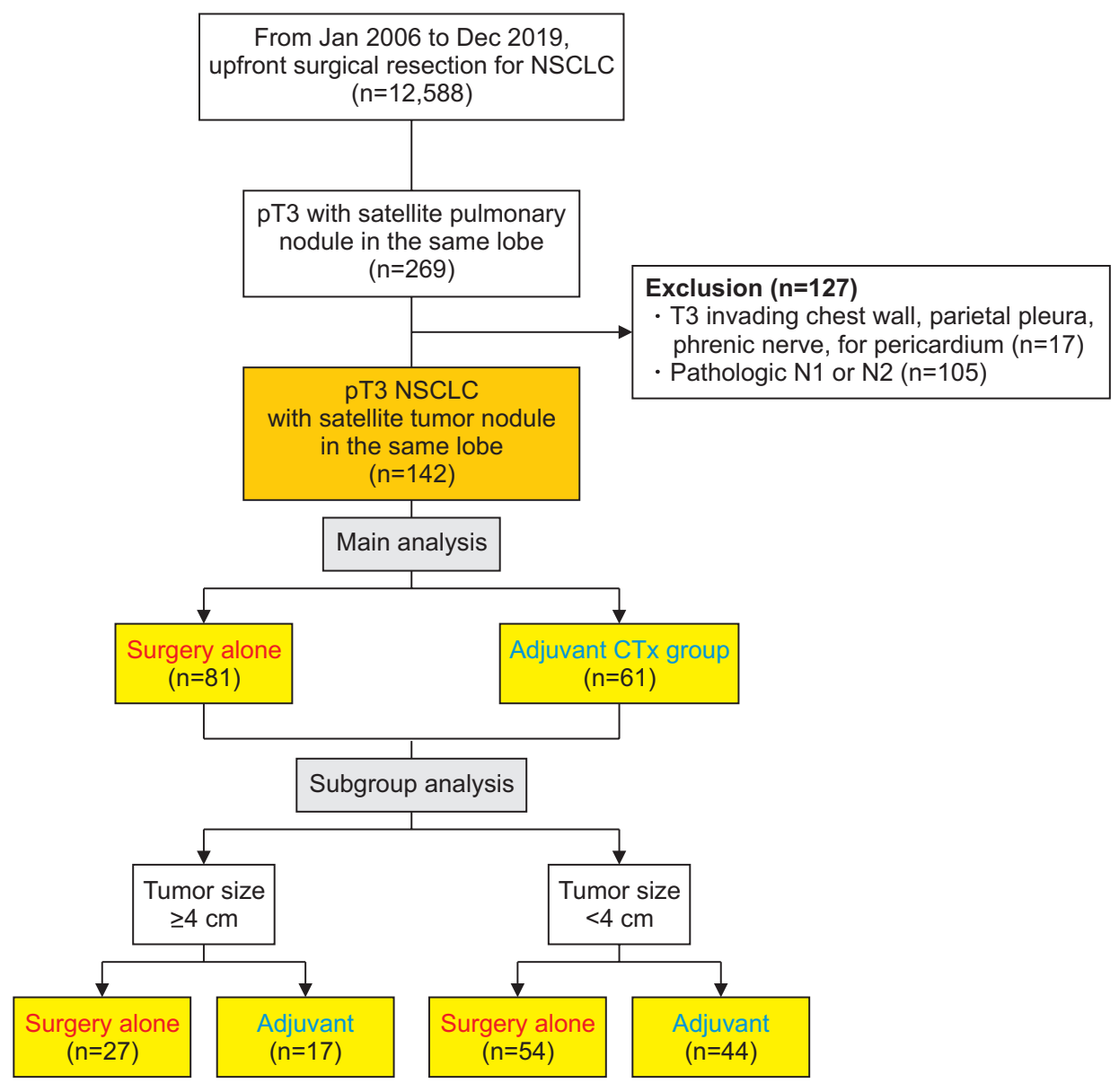

Fig. 1. Flow chart of study population. NSCLC, non-small-cell lung cancer; CTx, chemotherapy.
CT scans when recurrence was suspected. Locoregional recurrence was defined as that occurring within the ipsilateral hemithorax, including the pleura and mediastinal lymph nodes. Distant recurrence was defined as developing within the contralateral hemithorax or in a distant solid organ. Whenever recurrence was suspected, we tried to obtain histological or unequivocal radiological proof. For patients lost to follow-up, a telephone interview was conducted to determine late outcomes.

\section{Treatment groups and outcome measurements}

The adjuvant chemotherapy group included patients who completed 4 cycles of adjuvant chemotherapy within 2 months of surgical resection, but no radiotherapy. The surgery-only group included patients who underwent curative resection but did not receive adjuvant chemotherapy or did not complete 4 cycles of adjuvant chemotherapy.

The primary outcome was overall survival, where patients were followed from the date of surgery to the date of death from any cause or the last surveillance visit. The secondary outcome was disease-free survival, and recurrence or death prior to evidence of recurrence was considered as an event. The patients were followed from the date of surgery to the date of recurrence, death, or the last surveillance without evidence of recurrence, whichever came first.

\section{Statistical analysis}

Descriptive statistics were used to assess demographic characteristics and patient outcomes. Continuous data were expressed as median (interquartile range) and compared using the Mann-Whitney U test. Categorical data were expressed as counts and proportions and compared using the Fisher exact test. Hazard ratios (HRs) for death and recurrence were estimated using a Cox proportional hazards model. For both outcomes, multivariable HRs were adjusted for age at the time of surgery, sex, smoking status, histology, visceral pleural invasion, and tumor size. Additionally, subgroup analyses were performed according 
to tumor size: $<4 \mathrm{~cm}$ or $\geq 4 \mathrm{~cm}$. All analyses were per- tion for Statistical Computing, Vienna, Austria). A p-value formed using R Studio ver. 1.2.5 (RStudio, Boston, MA, $<0.05$ was considered to indicate statistical significance. USA) with R statistical language ver. 3.6.2 (The R Founda-

Table 1. Characteristics of the study population

\begin{tabular}{|c|c|c|c|c|}
\hline Characteristic & Total $(n=142)$ & Surgery-only group $(n=81)$ & Adjuvant group $(n=61)$ & p-value \\
\hline Age (yr) & $66.00(58.00-70.00)$ & $69.00(63.00-73.00)$ & $63.00(54.00-67.00)$ & $<0.001$ \\
\hline Sex & & & & 0.772 \\
\hline Female & $66(46.5)$ & $39(48.1)$ & $27(44.3)$ & \\
\hline Male & $76(53.5)$ & $42(51.9)$ & $34(55.7)$ & \\
\hline Charlson comorbidity index & & & & 0.945 \\
\hline 0 & $83(59.3)$ & $46(58.2)$ & $37(60.7)$ & \\
\hline 1 & $34(24.3)$ & $20(25.3)$ & $14(23.0)$ & \\
\hline 2 or more & $23(16.4)$ & $13(16.5)$ & $10(16.4)$ & \\
\hline Forced expiratory volume at 1 second (\%) & $94.00(82.00-105.50)$ & $91.00(81.00-103.50)$ & $96.00(85.75-107.00)$ & 0.296 \\
\hline Diffusing capacity of carbon monoxide (\%) & $87.00(75.00-97.00)$ & $87.00(72.00-96.00)$ & $86.50(76.25-97.75)$ & 0.756 \\
\hline Smoking & & & & 0.540 \\
\hline Never-smoker & $62(47.0)$ & $37(50.0)$ & $25(43.1)$ & \\
\hline Ever-smoker & $70(53.0)$ & $37(50.0)$ & $33(56.9)$ & \\
\hline Tumor laterality & & & & 0.919 \\
\hline Left & $61(43.0)$ & $34(42.0)$ & $27(44.3)$ & \\
\hline Right & $81(57.0)$ & $47(58.0)$ & $34(55.7)$ & \\
\hline Primary site & & & & 0.914 \\
\hline Lower & $57(41.3)$ & $32(41.6)$ & $25(41.0)$ & \\
\hline Middle & $12(8.7)$ & $6(7.8)$ & $6(9.8)$ & \\
\hline Upper & $69(50.0)$ & $39(50.6)$ & $30(49.2)$ & \\
\hline Clinical T category & & & & 0.251 \\
\hline T1a & $8(5.6)$ & $5(6.2)$ & $3(4.9)$ & \\
\hline $\mathrm{T} 1 \mathrm{~b}$ & $23(16.2)$ & $16(19.8)$ & $7(11.5)$ & \\
\hline $\mathrm{T} 1 \mathrm{c}$ & $33(23.2)$ & $15(18.5)$ & $18(29.5)$ & \\
\hline $\mathrm{T} 2 \mathrm{a}$ & $25(17.6)$ & $17(21.0)$ & $8(13.1)$ & \\
\hline $\mathrm{T} 2 \mathrm{~b}$ & $13(9.2)$ & $9(11.1)$ & $4(6.6)$ & \\
\hline T3 & $39(27.5)$ & $19(23.5)$ & $20(32.8)$ & \\
\hline $\mathrm{T} 4$ & $1(0.7)$ & 0 & $1(1.6)$ & \\
\hline Clinical $\mathrm{N}$ category & & & & 0.125 \\
\hline No & $130(91.5)$ & $71(87.7)$ & $59(96.7)$ & \\
\hline N1 & $9(6.3)$ & 7 (8.6) & $2(3.3)$ & \\
\hline N2 & $3(2.1)$ & $3(3.7)$ & 0 & \\
\hline Clinical stage group & & & & 0.500 \\
\hline IA1 & $8(5.6)$ & $5(6.2)$ & $3(4.9)$ & \\
\hline $\mathrm{IA} 2$ & $21(14.8)$ & $14(17.3)$ & $7(11.5)$ & \\
\hline $\mathrm{IA} 3$ & $31(21.8)$ & $14(17.3)$ & $17(27.9)$ & \\
\hline IB & $24(16.9)$ & $16(19.8)$ & $8(13.1)$ & \\
\hline IIA & $10(7.0)$ & $6(7.4)$ & $4(6.6)$ & \\
\hline IIB & $41(28.9)$ & $21(25.9)$ & $20(32.8)$ & \\
\hline IIIA & $5(3.5)$ & $4(4.9)$ & $1(1.6)$ & \\
\hline IIIB & $1(0.7)$ & $1(1.2)$ & 0 & \\
\hline IV & $1(0.7)$ & 0 & $1(1.6)$ & \\
\hline Histologic subtype & & & & 0.518 \\
\hline Adenocarcinoma & $94(66.2)$ & $52(64.2)$ & $42(68.9)$ & \\
\hline Squamous cell carcinoma & $34(23.9)$ & $19(23.5)$ & $15(24.6)$ & \\
\hline Others & $14(9.9)$ & $10(12.3)$ & $4(6.6)$ & \\
\hline
\end{tabular}


Table 1. Continued

\begin{tabular}{|c|c|c|c|c|}
\hline Characteristic & Total $(n=142)$ & Surgery-only group $(n=81)$ & Adjuvant group $(n=61)$ & $\mathrm{p}$-value \\
\hline Adenocarcinoma subtype & 94 & 52 & 42 & \\
\hline Acinar pattern & $38(40.4)$ & $20(38.5)$ & $18(42.9)$ & \\
\hline Papillary pattern & $26(27.7)$ & $15(28.8)$ & $11(26.2)$ & \\
\hline Mucinous & $9(9.6)$ & $5(9.6)$ & $4(9.5)$ & \\
\hline Lepidic pattern & $7(7.4)$ & $5(9.6)$ & $2(4.8)$ & \\
\hline Pleomorphic & $2(2.1)$ & 0 & $2(4.8)$ & \\
\hline Unknown & $12(12.8)$ & $7(13.5)$ & $5(11.9)$ & \\
\hline Tumor size $(\mathrm{mm})$ & $28.00(20.25-43.00)$ & $28.00(19.00-45.00)$ & $28.00(22.00-40.00)$ & 0.628 \\
\hline Extent of resection & & & & 0.049 \\
\hline Sublobar resection & $9(6.3)$ & $8(9.9)$ & $1(1.6)$ & \\
\hline Lobectomy & $127(89.4)$ & $68(84.0)$ & $59(96.7)$ & \\
\hline Sleeve resection & $6(4.2)$ & $5(6.2)$ & $1(1.6)$ & \\
\hline Total no. of removed lymph node & $17.00(11.27-24.00)$ & $17.00(9.01-23.00)$ & $17.00(14.00-24.00)$ & 0.316 \\
\hline Tumor differentiation & & & & 0.934 \\
\hline G1 & $16(11.3)$ & $8(9.9)$ & $8(13.1)$ & \\
\hline G2 & $86(60.6)$ & $50(61.7)$ & $36(59.0)$ & \\
\hline G3 & $25(17.6)$ & $14(17.3)$ & $11(18.0)$ & \\
\hline Gx & $15(10.6)$ & $9(11.1)$ & $6(9.8)$ & \\
\hline Resection margin & & & & $>0.999$ \\
\hline Ro & $140(98.6)$ & $80(98.8)$ & $60(98.4)$ & \\
\hline R1 & $2(1.4)$ & $1(1.2)$ & $1(1.6)$ & \\
\hline Lymphovascular invasion & $38(29.2)$ & $23(38.3)$ & $15(21.4)$ & 0.055 \\
\hline Pleural invasion & & & & 0.987 \\
\hline PLO & $119(89.5)$ & $68(89.5)$ & $51(89.5)$ & \\
\hline PL1 & $9(6.8)$ & $5(6.6)$ & $4(7.0)$ & \\
\hline PL2 & $5(3.8)$ & $3(3.9)$ & $2(3.5)$ & \\
\hline Hospital stay (day) & $6(5-9)$ & $7(5-10)$ & $6(5-8)$ & 0.250 \\
\hline Clavien-Dindo classification, III or greater & $43(30.3)$ & $29(35.8)$ & $14(23.0)$ & 0.143 \\
\hline Adjuvant chemotherapy & & & & $<0.001$ \\
\hline Complete adjuvant therapy & $61(43.0)$ & 0 & $61(100.0)$ & \\
\hline Incomplete adjuvant therapy & $13(9.2)$ & $13(16.0)$ & 0 & \\
\hline No adjuvant therapy & $68(47.9)$ & $68(84.0)$ & 0 & \\
\hline
\end{tabular}

Values are presented as median (interquartile range) or number (\%).

\section{Results}

\section{Patient characteristics}

The median age of the 142 patients was 66 years (interquartile range [IQR], 58-70 years), and $53.2 \%(\mathrm{n}=74)$ were men. Adjuvant chemotherapy was completed in 61 patients (43.9\%, adjuvant group). Patients who did not complete adjuvant chemotherapy $(n=13,9.7 \%)$ and did not undergo adjuvant therapy ( $\mathrm{n}=68,47.9 \%)$ were classified as belonging to the surgery-only group $(\mathrm{n}=81)$ (Table 1$)$. The reasons for not completing adjuvant therapy in 13 patients were as follows: toxicity during chemotherapy $(\mathrm{n}=5)$; poor performance status after surgery $(n=3)$; postoperative complications such as pulmonary embolism, pneumonia, and pleural effusion ( $\mathrm{n}=3)$; and unknown reasons $(\mathrm{n}=2)$ (Table 2).

The adjuvant group was associated with a younger age. However, there were no significant differences in baseline characteristics, including smoking history, Charlson comorbidity index, preoperative forced expiratory volume at 1 second, and diffusing capacity of carbon monoxide across between the 2 groups. The surgical and tumor characteristics were also similar between the 2 groups. In both groups, most tumors were clinical T1 or T2 and adenocarcinoma, and most patients underwent lobectomy. The median tumor size was $28 \mathrm{~mm}$ (IQR, 22-40 $\mathrm{mm}$ ) in the adjuvant group and $28 \mathrm{~mm}$ (IQR, 19-45 $\mathrm{mm}$ ) in the surgery-only group ( $\mathrm{p}=0.628$ ). The median hospital stay for patients in the adjuvant group and surgery-only group was 6 days (IQR, $5-8$ days) and 7 days (IQR, $5-10$ days) $(\mathrm{p}=0.250$ ), re- 
spectively. The incidence of major postoperative complications (Clavien-Dindo classification III or greater) was observed in 43 out of 142 patients (30.3\%), with no significant difference between the 2 groups (surgery-only group $35.8 \%$ versus adjuvant group $23.0 \%, \mathrm{p}=0.143$ ).

\section{Survival outcomes}

During a median follow-up of 48.8 months (IQR, 28.885.7 months), the adjuvant group and surgery-only group had 12 and 29 deaths, and 14 and 26 recurrences, respectively. The overall 5-year survival for patients in the adjuvant group and the surgery-only group was $80.8 \%$ and $64.6 \%(\mathrm{p}=0.024)$, and the 5 -year disease-free survival rate was $76.4 \%$ and $54.2 \%(p=0.018)$, respectively (Fig. 2). In the multivariable Cox proportional analysis, the use of adjuvant chemotherapy was associated with better overall survival than resection alone (adjusted hazard ratio, 0.47; 95\% confidence interval, 0.23-0.95; $\mathrm{p}=0.035$ ) (Fig. 3).

Table 2. Reason for incomplete chemotherapy

\begin{tabular}{lc}
\hline \multicolumn{1}{c}{ Reason for incomplete chemotherapy } & No. \\
\hline Total & 13 \\
Poor performance status & 3 \\
Chemotherapy toxicity & 5 \\
Acute pulmonary embolism & 1 \\
Pneumonia & 1 \\
Pleural effusion & 1 \\
Unknown reasons & 2 \\
\hline
\end{tabular}

A

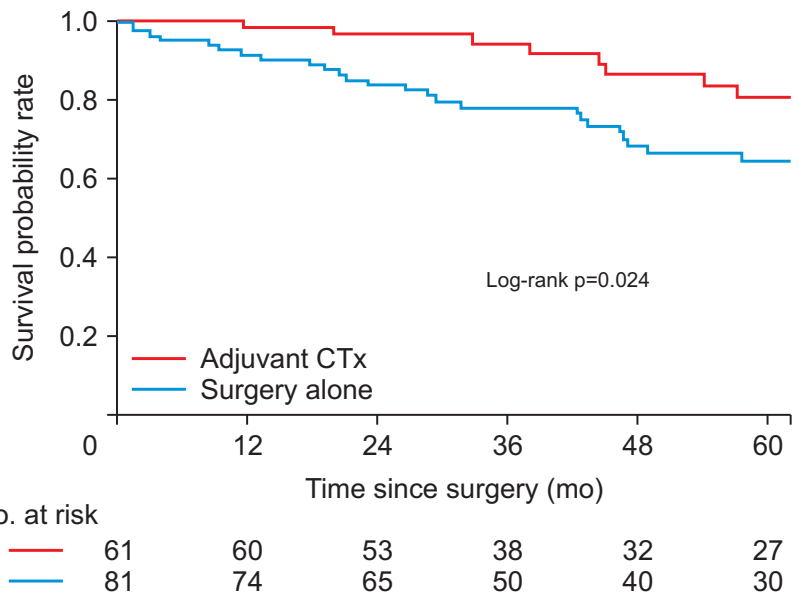

\section{Subgroup analysis by tumor size}

In the adjuvant and surgery-only groups, there were 17 and 27 patients with tumors $4 \mathrm{~cm}$ or greater in size, and 44 and 54 patients with tumors smaller than $4 \mathrm{~cm}$, respectively. Among the patients with a tumor size of $4 \mathrm{~cm}$ or greater, the 5 -year overall survival rate for patients in the adjuvant group and surgery-only group was $91.7 \%$ and $37.5 \%$ $(\mathrm{p}=0.003)$, and the 5 -year disease-free survival rate was $88.2 \%$ and $23.3 \%(\mathrm{p}<0.001)$, respectively (Fig. 4 A, B). However, in patients with tumors smaller than $4 \mathrm{~cm}$, there were no significant differences in overall survival $(\mathrm{p}=0.430)$ and disease-free survival $(p=0.610)$ between the adjuvant group and surgery-only group (Fig. 4C, D).

\section{Recurrence patterns}

During a median surveillance follow-up of 18.1 months (IQR, 8.7-39.4 months), there were 40 recurrences: $23 \%$ in the adjuvant group and $32.1 \%$ in the surgery-only group $(\mathrm{p}=0.312)$. Distant failure was the most common pattern of recurrence in both groups.

\section{Discussion}

In this study, we demonstrated a survival benefit associated with adjuvant chemotherapy for patients with pathologic N0 disease with a satellite pulmonary nodule in the same lobe. Our study showed a $16.2 \%$ improvement in overall survival at 5 years after receiving adjuvant chemotherapy, which is consistent with a randomized controlled trial in Canada (JBR. 10 trial) [6]. This survival advantage

B

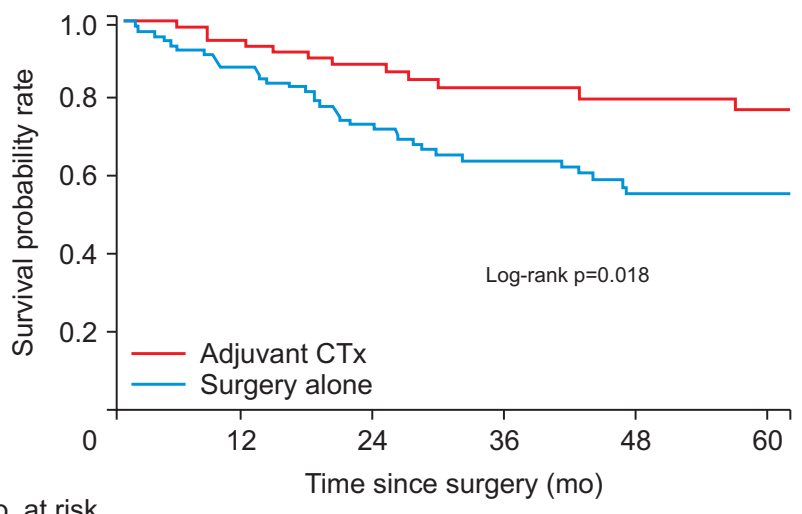

No. at risk

$\begin{array}{lllll}57 & 48 & 31 & 27 & 23 \\ 71 & 55 & 38 & 29 & 23\end{array}$

Fig. 2. Kaplan-Meier estimates of overall (A) and disease-free survival (B) comparing the adjuvant group with the surgery-only group. CTx, chemotherapy. 


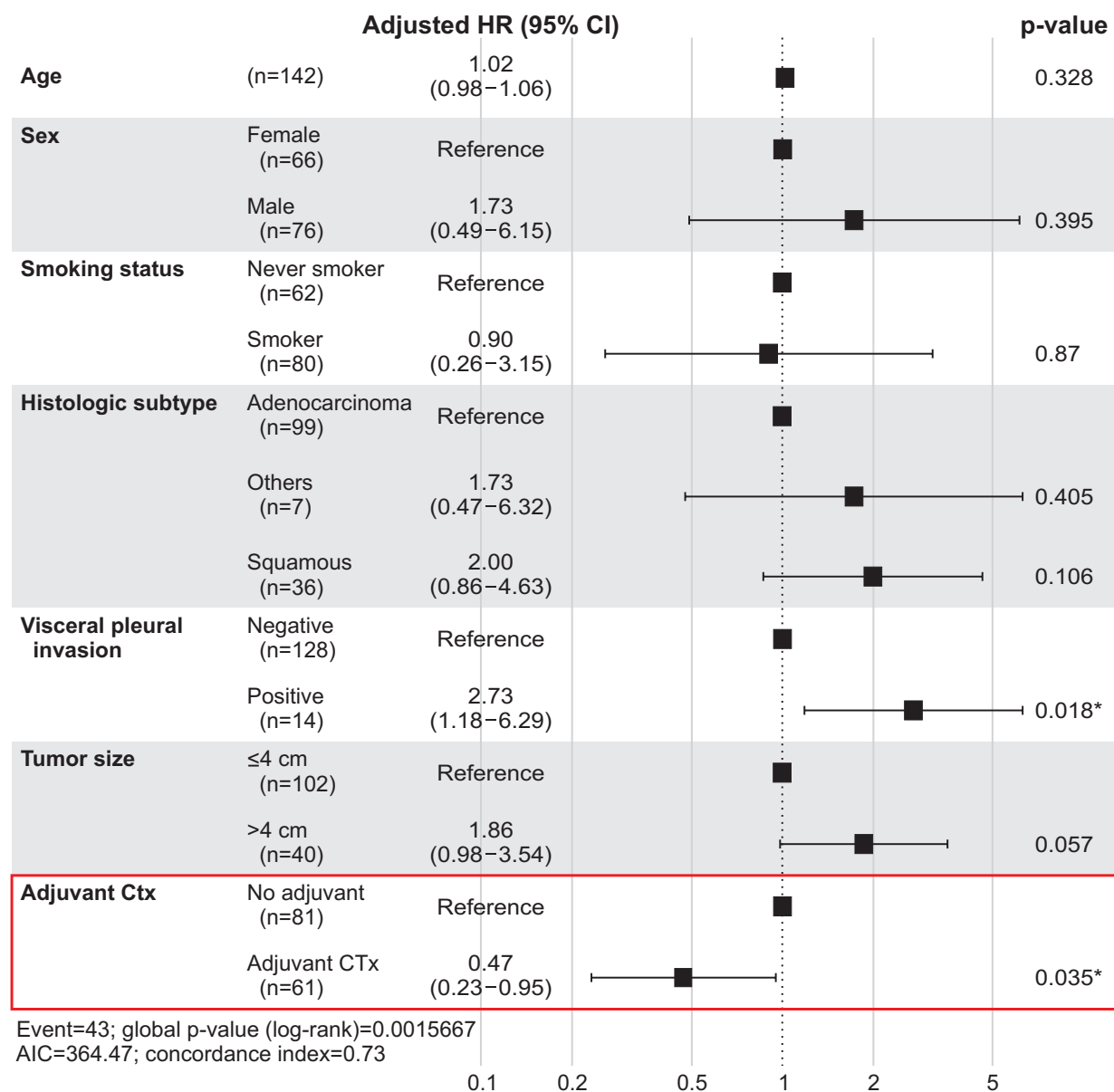

Fig. 3. Multivariable Cox proportional hazard model for overall survival. $\mathrm{HR}$, hazard ratio; $\mathrm{Cl}$, confidence interval; CTx, chemotherapy; AIC, Akaike information criterion. ${ }^{*} \mathrm{p}<0.05$ (statistically significant). of adjuvant chemotherapy use was significant in the subset of node-negative patients with a tumor size of $4 \mathrm{~cm}$ or greater. However, there were no significant differences between the adjuvant group and the surgery-only group in the subpopulation of patients with node-negative disease and tumors smaller than $4 \mathrm{~cm}$. In the multivariable analysis, adjuvant chemotherapy was not an independent factor for overall survival and disease-free survival.

In a previous study, the reported 5-year overall survival rate averaged $40 \%$ (range, 20\%-64\%) [14] after surgical resection of a satellite nodule in the same lobe, which falls within the range of 5 -year overall survival of the T3 subset (41\%-49\%) [15]. As a result, this staging parameter was moved from T4 to T3 in the seventh edition of the American Joint Committee on Cancer staging manual [15]. However, it remains unclear whether adjuvant therapy is necessary for this cohort. Given that platinum-based adjuvant chemotherapy has shown a survival benefit of $5.4 \%$ in node-positive stage II and III NSCLCs in multiple clinical trials [2], the use of adjuvant chemotherapy should be considered for node-positive cases with a satellite pulmonary nodule. However, there is limited evidence supporting the use of adjuvant chemotherapy in node-negative NSCLC with satellite pulmonary nodule in the same lobe. Since this subgroup represents a small fraction of the NSCLC population, these patients have not been extensively studied.

In our cohort, the incidence of node-negative satellite pulmonary nodule without other invasive T3 characteristics was $21 \%$. Most of them $(72 \%, n=102)$ were associated with tumors smaller than $4 \mathrm{~cm}$. Satellite pulmonary nodules were found only after resection in a considerable proportion of patients: $72 \%$ of the patients were clinically classified as T1 or T2, although they were pathological stage T3, which is consistent with other reports [16,17]. In an intention-to-treat analysis, patients who did not complete adjuvant therapy also must be included in the treatment arm. However, in the literature, these patients showed worse survival than patients who completed adjuvant therapy. We also observed in our preliminary analysis that the survival of these patients was similar to that of patients who did not receive adjuvant therapy (Supplementary Figs. 1, 2). 
A Overall survival, size $\geq 4 \mathrm{~cm}$

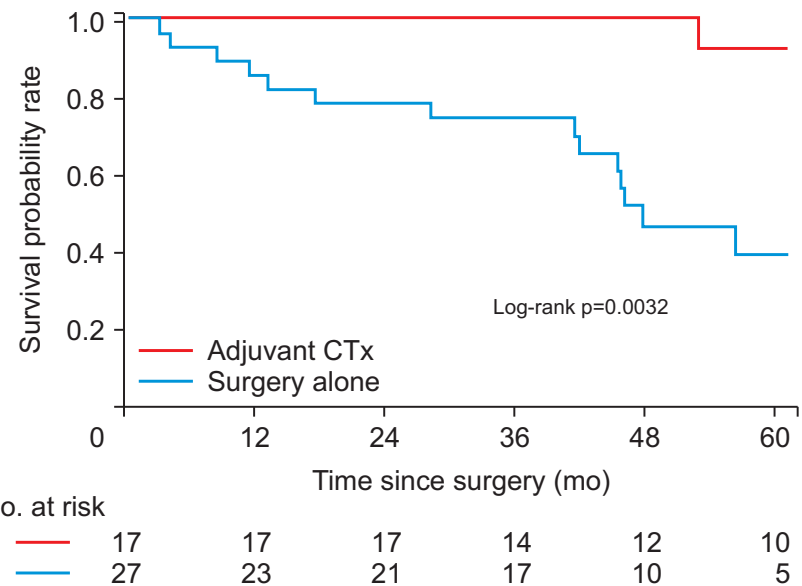

C Overall survival, size $<4 \mathrm{~cm}$

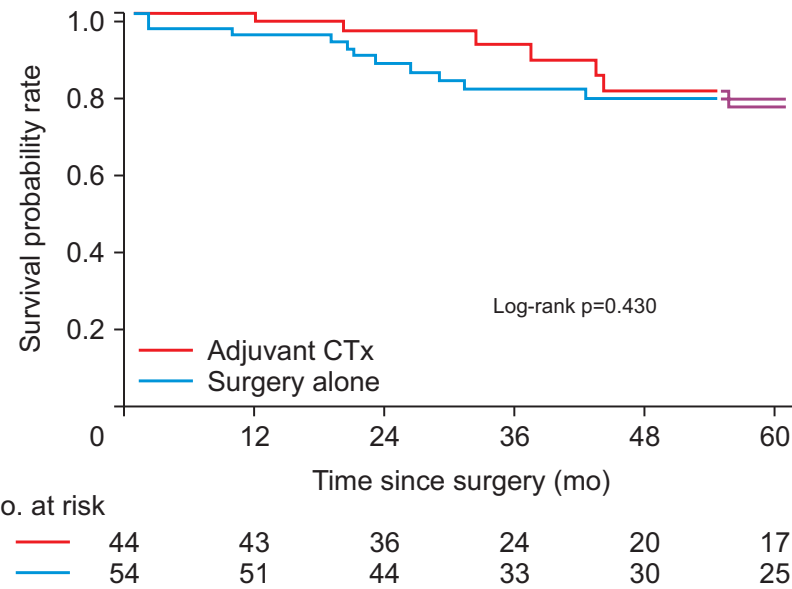

B Disease-free survival, size $\geq 4 \mathrm{~cm}$

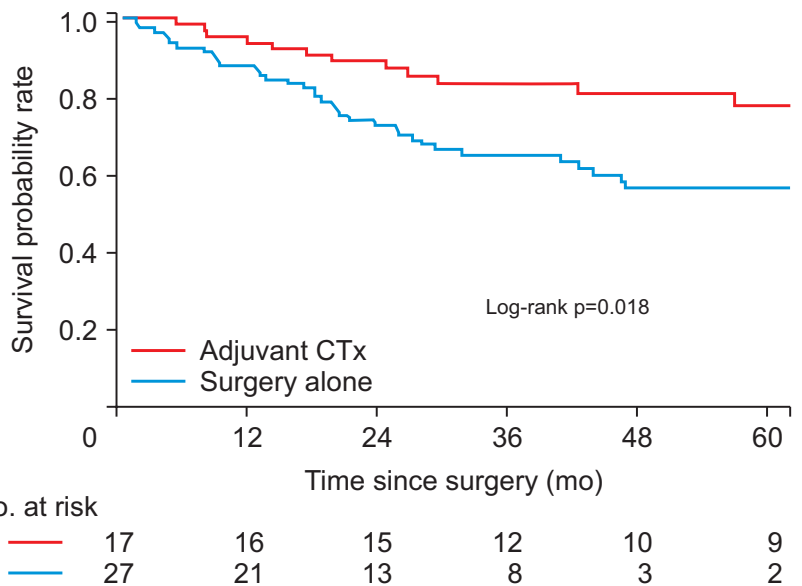

D Disease-free survival, size $<4 \mathrm{~cm}$

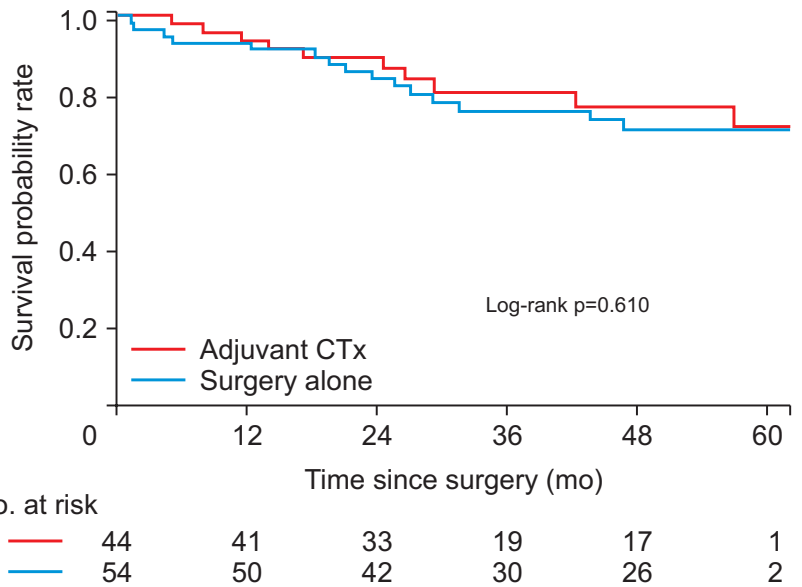

Fig. 4. Kaplan-Meier estimates of overall and disease-free survival comparing the adjuvant group with the surgery-only group for the subgroups of tumors $4 \mathrm{~cm}$ or greater in size (A, B) and tumors smaller than $4 \mathrm{~cm}(C, D)$.

These observations also drove us to design a per-protocol analysis and include patients who did not complete adjuvant therapy in the surgery-only group. We believe that the interpretation of the study is not biased by the treatment grouping, since only patients who completed the planned cycle benefited from adjuvant therapy. Furthermore, there were no demonstrable differences in clinicopathological characteristics between the 2 groups regarding hospital mortality and postoperative complications. This indicates that the 2 groups constituted a well-defined counterfactual model and the use of adjuvant therapy was solely at the discretion of the physician.

In the present study, we demonstrated the survival benefit associated with the use of adjuvant therapy in patients with node-negative NSCLC with satellite pulmonary nodules in the same lobe. This finding is in line with the latest retrospective study in the United States using the NCDB
[12]. In that study, the authors found an absolute improvement of $11 \%$ in 3 -year overall survival associated with the use of adjuvant therapy in patients with T3 NSCLC and an additional nodule in the same lobe. Furthermore, the authors showed a survival benefit of adjuvant therapy in node-negative patients with tumors smaller than $4 \mathrm{~cm}$. Consistent with these results, in our study, these survival benefits of adjuvant chemotherapy were evident in a subset of node-negative patients with a tumor size of $4 \mathrm{~cm}$ or greater. However, we did not find a statistically significant difference between the adjuvant group and surgery-only group in the subgroup of patients with node-negative disease and tumors smaller than $4 \mathrm{~cm}$.

The failure of adjuvant therapy to improve survival in our study for tumors smaller than $4 \mathrm{~cm}$ can be explained for several reasons. First, our study was limited by the relatively small number of patients, which may have limited its 
ability to detect small differences in survival (i.e., type II error). However, the retrospective US study using the NCDB may have been confounded by potential unmeasured differences in characteristics, as the data collected from the NCDB did not include important variables such as pulmonary function testing [12]. In contrast, in our study, we sufficiently reviewed the variables influencing treatment and survival outcomes. Therefore, our findings may reflect real-world results. More studies are needed to explore whether the use of adjuvant therapy improves survival outcomes. The use of adjuvant therapy should be supported by the underlying mechanism, but the mechanisms responsible for these satellite pulmonary nodules are unclear. In the past, satellite nodules were explained by the result of peripheral embolization through the pulmonary or lymphatic vessels [18], as well as the manifestation of "field cancerization"- that is, a field effect in which most of the respiratory epithelium mutated due to exposure to carcinogens [19]. Interestingly, $29.2 \%$ of the patients in our study had lymphovascular invasion. This finding implies that microscopic metastasis may persist even after curative resection. With the current result that metastasis accounted for the majority of cases of recurrence, these findings may collectively support the use of adjuvant chemotherapy. Further investigations are needed to investigate the underlying mechanism of development of satellite pulmonary nodules.

Our study has several limitations. Due to the observational nature of this study, there may have been residual confounding. However, we included various sociodemographic and clinical characteristics that are potential confounders. Additionally, it is possible that our satellite pulmonary nodule cohort may have included patients with synchronous multiple primary lung cancer. This category is increasing in frequency, but is less likely to be associated with nodal metastasis or systemic metastases. To reduce misclassification as much as possible, we thoroughly reviewed the histology and immunochemistry results within pathologic data in patients with multiple primary lung cancer and satellite pulmonary nodules. Then, the presence of a satellite pulmonary nodule was determined based on preoperative imaging and the surgeon's decision. Nonetheless, certain characteristics of satellite nodules, such as number and size, might contribute to the patient's survival outcome and the effect of adjuvant therapy. However, this information was not evaluated in the pathologic report, and we could not include these factors in the analysis. Finally, since our data were obtained from a single tertiary-referral academic center, it may be difficult to generalize our findings. Further validation studies with multiple institutions are required.

In conclusion, adjuvant chemotherapy was associated with increased overall and disease-free survival in T3 cancers with additional nodules in the same lobe. This survival benefit was significant in node-negative tumors $4 \mathrm{~cm}$ or greater in size, but not in tumors smaller than $4 \mathrm{~cm}$. The current study supports the use of adjuvant chemotherapy in this patient subpopulation, especially in the presence of a tumor $4 \mathrm{~cm}$ or greater in size. Additional studies are required to refine the role of adjuvant chemotherapy for tumors smaller than $4 \mathrm{~cm}$ with satellite pulmonary nodules.

\section{Conflict of interest}

No potential conflict of interest relevant to this article was reported.

\section{ORCID}

Jiyoun Park: https://orcid.org/0000-0002-6668-1406 Junghee Lee: https://orcid.org/0000-0003-0466-7630 Yeong Jeong Jeon: https://orcid.org/0000-0001-6745-6131 Sumin Shin: https://orcid.org/0000-0002-8986-6622 Jong Ho Cho: https://orcid.org/0000-0003-3362-4621 Hong-Kwan Kim: https://orcid.org/0000-0002-7815-3336 Yong Soo Choi: https://orcid.org/0000-0001-8492-7644 Jhingook Kim: https://orcid.org/0000-0002-3828-0453 Jae Ill Zo: https://orcid.org/0000-0002-0996-1612

Young Mog Shim: https://orcid.org/0000-0001-5924-9765

\section{Supplementary materials}

Supplementary materials can be found via https://doi. org/10.5090/jcs.21.110. Supplementary Fig. 1. Overall and recurrence-free survival comparing the adjuvant-complete group, adjuvant-incomplete group, and the surgery-only group. Supplementary Fig. 2. Overall and recurrence-free survival comparing the adjuvant-complete group, adjuvant-incomplete group, and surgery-only group in nonsmall-cell lung cancer patients with node-negative disease and tumors smaller than $4 \mathrm{~cm}$.

\section{References}

1. Choi YS, Shim YM, Kim K, Kim J. Pattern of recurrence after curative resection of local (stage I and II) non-small cell lung cancer: difference according to the histologic type. J Korean Med Sci 2004;19: 674-6. 
2. Pignon JP, Tribodet H, Scagliotti GV, et al. Lung adjuvant cisplatin evaluation: a pooled analysis by the LACE Collaborative Group. J Clin Oncol 2008;26:3552-9.

3. Scagliotti GV, Fossati R, Torri V, et al. Randomized study of adjuvant chemotherapy for completely resected stage I, II, or IIIA nonsmall-cell lung cancer. J Natl Cancer Inst 2003;95:1453-61.

4. Waller D, Peake MD, Stephens RJ, et al. Chemotherapy for patients with non-small cell lung cancer: the surgical setting of the Big Lung Trial. Eur J Cardiothorac Surg 2004;26:173-82.

5. Arriagada R, Bergman B, Dunant A, et al. Cisplatin-based adjuvant chemotherapy in patients with completely resected non-small-cell lung cancer. N Engl J Med 2004;350:351-60.

6. Winton T, Livingston R, Johnson D, et al. Vinorelbine plus cisplatin vs. observation in resected non-small-cell lung cancer. N Engl J Med 2005;352:2589-97.

7. Douillard JY, Rosell R, De Lena M, et al. Adjuvant vinorelbine plus cisplatin versus observation in patients with completely resected stage IB-IIIA non-small-cell lung cancer (Adjuvant Navelbine International Trialist Association [ANITA]): a randomised controlled trial. Lancet Oncol 2006;7:719-27.

8. Postmus PE, Kerr KM, Oudkerk M, et al. Early and locally advanced non-small-cell lung cancer (NSCLC): ESMO Clinical Practice Guidelines for diagnosis, treatment and follow-up. Ann Oncol 2017; 28(suppl_4):iv1-21.

9. Howington JA, Blum MG, Chang AC, Balekian AA, Murthy SC. Treatment of stage I and II non-small cell lung cancer: diagnosis and management of lung cancer, 3rd ed: American College of Chest Physicians evidence-based clinical practice guidelines. Chest 2013;143(5 Suppl):e278S-e313S.

10. Kris MG, Gaspar LE, Chaft JE, Kennedy EB. Adjuvant systemic therapy and adjuvant radiation therapy for stages I to IIIA resectable non-small-cell lung cancers: American Society of Clinical Oncology/
Cancer Care Ontario Clinical Practice Guideline update summary. J Oncol Pract 2017;13:449-51.

11. Strauss GM, Herndon JE 2nd, Maddaus MA, et al. Adjuvant paclitaxel plus carboplatin compared with observation in stage IB nonsmall-cell lung cancer: CALGB 9633 with the Cancer and Leukemia Group B, Radiation Therapy Oncology Group, and North Central Cancer Treatment Group Study Groups. J Clin Oncol 2008;26:504351 .

12. Salazar MC, Rosen JE, Arnold BN, et al. Adjuvant chemotherapy for T3 non-small cell lung cancer with additional tumor nodules in the same lobe. J Thorac Oncol 2016;11:1090-100.

13. Jairam V, Park HS. Strengths and limitations of large databases in lung cancer radiation oncology research. Transl Lung Cancer Res 2019;8(Suppl 2):S172-83.

14. Kim AW, Cooke DT. Additional pulmonary nodules in the patient with lung cancer: controversies and challenges. Clin Chest Med 2011;32:811-25.

15. Goldstraw P, Crowley J, Chansky K, et al. The IASLC Lung Cancer Staging Project: proposals for the revision of the TNM stage groupings in the forthcoming (seventh) edition of the TNM Classification of malignant tumours. J Thorac Oncol 2007;2:706-14.

16. Rao J, Sayeed RA, Tomaszek S, Fischer S, Keshavjee S, Darling GE. Prognostic factors in resected satellite-nodule T4 non-small cell lung cancer. Ann Thorac Surg 2007;84:934-9.

17. Port JL, Korst RJ, Lee PC, Kansler AL, Kerem Y, Altorki NK. Surgical resection for multifocal (T4) non-small cell lung cancer: is the T4 designation valid? Ann Thorac Surg 2007;83:397-400.

18. Urschel JD, Urschel DM, Anderson TM, Antkowiak JG, Takita H. Prognostic implications of pulmonary satellite nodules: are the 1997 staging revisions appropriate? Lung Cancer 1998;21:83-7, 89-91.

19. Johnson BE. Second lung cancers in patients after treatment for an initial lung cancer. J Natl Cancer Inst 1998;90:1335-45. 